\title{
Malaria and the liver: exploring the silent pathway
}

The circumsporozoite protein (CSP) has a key role in several stages of the Plasmodium life cycle, including the development of the sporozoite form of the parasite and its entry into the salivary glands of the mosquito vector. Now, a new role has been discovered for this surfaceexpressed, multifunctional protein in the under-explored liver stage of the parasite. Reporting in Cell, Singh and colleagues show that by altering the expression of over 1,000 host genes, CSP creates a favourable environment that facilitates the growth and development of the parasite in liver hepatocytes.

Malaria infection is initiated by the injection of Plasmodium sporozoites from infected mosquitoes into the skin of the mammalian host. The parasite then enters the circulatory system and quickly travels to the liver. An obligatory step during the malaria infection cycle is the development of Plasmodium sporozoites into thousands of merozoites in each hepatocyte. This development process occurs within the cytoplasm of the
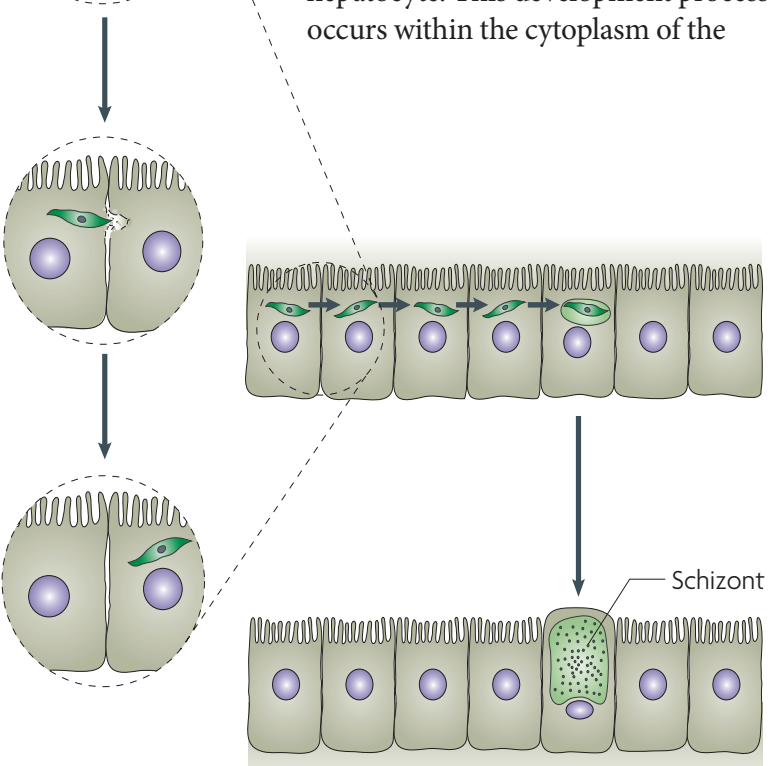

After the Plasmodium sporozoite (green) enters a hepatocyte, it traverses several hepatocytes until it becomes established in one cell, in which a PV is formed. Each invading sporozoite develops and multiplies inside a hepatocyte, forming

thousands of merozoites. Image reproduced, with permission, from Prudencio, M. et al. Nature Reviews Microbiology @ (2006) Macmillan Publishers Ltd. hepatocyte in a parasitophorous vacuole $(\mathrm{PV})$. Following release from hepatocytes, each merozoite infects an erythrocyte, thus triggering the bloodstream stage of infection and the pathology that is associated with malaria.

\section{C....CSP creates a} favourable environment that facilitates the growth and development of the parasite in liver hepatocytes.

Earlier research had revealed that following hepatocyte invasion, CSP gains access to the cytoplasm and becomes associated with the nucleus of the cell. To investigate the role of CSP in the liver stage of malaria infection, the authors set out to investigate how the protein escapes the $\mathrm{PV}$, which is normally impermeable to macromolecules. Their analysis revealed that traversal of CSP across the $\mathrm{PV}$ membrane is mediated by amino-terminal pexel/vacuolar transport signal (VTS) motifs, a well-characterized signal sequence that mediates protein export. In addition, the authors demonstrated that CSP contains a functional nuclear localization signal and that entry of the protein into the nucleus is mediated by the importin $\alpha 3 / \beta 1$. As nuclear factor (NF)- $\mathrm{kB}$ - a host transcription factor that controls the expression of many of the genes that are involved in the host immune response - also uses the importin $\alpha 3$ pathway, Singh et al. postulated that CSP interferes with NF- $\kappa \mathrm{B}$ translocation into the nucleus and, therefore, inhibits the host response that is mediated by this factor. Using various experimental approaches, the authors confirmed that CSP does inhibit NF- $\mathrm{BB}$ nuclear import and the expression of many of the genes that are controlled by this transcription factor. Indeed, microarray analysis revealed that the expression of over 1,100 genes that are involved in a diverse range of biological processes were influenced by the presence of CSP. So, having established that CSP escapes from the PV into the cytoplasm and then enters the nucleus, what impact does the protein have on liver-stage sporozoite biology? Using pexel/VTS mutant parasites to address this question, it was shown that functional CSP significantly enhances parasite growth and development. This allowed the authors to conclude that the protein contributes to creating a hospitable environment for Plasmodium during that crucial stage of infection between the bite of a parasite-infected mosquito and the release of merozoites into the bloodstream.

As sporozoite multiplication in the liver is not associated with pathology, this stage of malaria infection is not a target for therapy. However, it is an attractive target for vaccine and prophylaxis strategies, as blocking infection at this stage will prevent pathology and disease. By building on the progress that is described in this study and gaining a full understanding of the molecular mechanisms that underlie parasite development in the liver, the goal of a new class of therapies directed at liver-stage malaria infection should become a reality.

David O’Connell

ORIGINAL RESEARCH PAPER Singh, A. P. et al. Plasmodium circumsporozoite protein promotes the development of the liver stages of the parasite. Cell 131, 492-504 (2007)

FURTHER READING Prudencio, M., Rodriguez, A \& Mota, M. M. The silent path to thousands of merozoites: the Plasmodium liver stage. Nature Rev. Microbiol. 4, 849-856 (2006) 